\title{
Psychological and Physiological Relaxation Induced by Nature-Working with Ornamental Plants
}

\author{
Jiang Tao, ${ }^{1}$ Ahmad Hassan $\left(\mathbb{D},{ }^{2}\right.$ Chen Qibing $\left(\mathbb{D},{ }^{2}\right.$ Liu Yinggao $\left(\mathbb{D},{ }^{3}\right.$ Guo Li,${ }^{2}$ Mingyan Jiang, \\ Deng Li, ${ }^{2}$ Li Nian, ${ }^{2}$ Lv Bing-Yang, ${ }^{2}$ and Zhong Ziqin ${ }^{4}$ \\ ${ }^{1}$ College of Forestry, Guizhou University, Guiyang, Guizhou 550025, China \\ ${ }^{2}$ College of Landscape Architecture, Sichuan Agricultural University, Chengdu, Sichuan 611130, China \\ ${ }^{3}$ College of Forestry, Sichuan Agricultural University, Chengdu, Sichuan 611130, China \\ ${ }^{4}$ College of Horticultural Sciences, Sichuan Agricultural University, Chengdu, Sichuan 611130, China
}

Correspondence should be addressed to Ahmad Hassan; ahmaduaf1416@gmail.com, Chen Qibing; 2948642202@qq.com, and Liu Yinggao; 499130793@qq.com

Received 24 February 2019; Accepted 30 September 2019; Published 7 March 2020

Academic Editor: Cengiz Çinar

Copyright (c) 2020 Jiang Tao et al. This is an open access article distributed under the Creative Commons Attribution License, which permits unrestricted use, distribution, and reproduction in any medium, provided the original work is properly cited.

\begin{abstract}
Objective. Nature has a significant impact on general well-being. However, till date, little psychophysiological evidence is available on the benefits of nature-based activities in adults. The primary goal of this study was to determine the physiological and psychological benefits of horticultural activity in adults. The participants were instructed to perform the making of a flower basket (horticultural activity) and a computer task (i.e., control activity) was compared. Methods. A total of 40 Chinese females (mean age $22.2 \pm 0.9$ years) participated in this experiment. The Blood pressure, the State-Trait Anxiety Inventory (STAI), and the electroencephalograms (EEGs) were used to measure the participants' psychophysiological responses. Results. Analysis of the STAI data showed a lower anxiety score after performing the horticultural activity than after performing the control activity. Furthermore, in the EEG evaluation, variations in the brainwaves were observed after both activities. Conclusions. The study results suggest that horticultural activity induced physiological and psychological relaxation in adults.
\end{abstract}

\section{Introduction}

Humans are considered to be entirely dependent on nature for their emotional, cognitive, and physical needs [1-3]. However, whether nature is beneficial for mental health remains under investigation by scientists at many educational institutions [4]. In the 1970s, scholars investigated the psychological role of nature applied psychometric measures to explore the eco-friendly perceptions and cognitive states of human subjects in natural surroundings $[5,6]$. However, in the 1970s, some scholars began to examine the effects of nature on the participants' psychological and "biological responses to various environments" [7-10]. Studies have found that contact with plants can have both psychological and physiological benefits $[7,11,12]$. Furthermore, involvement in nature and/ or wilderness settings can help with stress management and can be healing [13]. In addition, humans who spend time in natural environments are healthier and more mentally active
[14]. Currently, people are increasingly living in urban places and spending more time indoors away from nature [15]. Many mental health problems, such as depression and stress, are believed to occur from a lack of exposure to nature or from living in an artificial world [16]. Furthermore, recent advancements in technology are also considered to be hurdles because most adult populations are connected to electronics, such as mobile phones and tablet devices, and they are losing their connection to the natural environment. Moreover, this dispersal of information technology causes considerable stress, such as technostress [17], which is a new disease of adaptation that is caused by an inability to cope with new computer technologies in a healthy way. Therefore, there is a growing need to reconnect adults to the healing powers of natural environments. People also receive benefits from plants in natural or vegetative surroundings in both direct and indirect ways such as lower blood pressure, increased productivity and attentiveness, reduced mental fatigue, and stress and fewer reports of 
illness [18]. Active contacts such as contact with plants have been shown to provide psychophysiological benefits, including improved social interaction, increased self-esteem, and reduced stress levels [5]. Limited numbers of studies have described the clinical effects of horticultural therapy; although, interactions with nature have been shown to have physical, emotional, social, and cognitive benefits that help reduce stress [19]. Few studies have examined the effects of indoor plants that are relevant to the well-being and effectiveness of office workers. These outcomes include task performance, room assessments psychophysiological stress responses, and emotional states [20-23]. Additionally, some experiments have explored attitudes towards plants in the office [24], and the effects of plants on the discomfort and health symptoms that are associated with sick building syndrome [25-27]. However, the findings from experiments that were conducted in either simulated or laboratory settings may not generalize well to real office settings. Additionally, to date, there is a lack of scientific research on EEG regarding the effects of plants in adults.

\section{Overview of Electroencephalograms (EEG) and Brainwaves}

Richard Caton (1875) discovered the existence of electrical currents in the brain. Subsequently, in 1924, Hans Berger was the first to record the electrical currents inside the brain. He used EEGs to explain electrical brain activity and discovered the alpha wave $(8-12 \mathrm{~Hz})$, which is also known as Berger's wave [28]. In 1934, Adrian and Matthews introduced new ideas regarding human brainwaves. During the 1950's, EEG technology was successfully applied in all fields of science including neurosurgery, neurology, and cognitive science. Finally, in 1964, Dr. Gray Walter connected electrodes directly to the motor areas of a human brain to investigate brainwave activity. The human brain consists of hundreds of billions of brain cells that are called neurons. These neurons have axons that can transmit signals through neurotransmitters to the dendrites of other neurons, which then lead to an electrical polarity change inside the neuronal axon of the receiving neuron. This polarity change can be recorded by using EEGs $[29,30]$. The EEG signals originate from the inhibitory (hyperpolarizing) and excitatory (depolarizing) post-synaptic potentials of the pyramidal neurons, which are present in the lower portion of the cerebral cortex [31, 32]. EEGs represent the electrical activity inside the brain and provide valuable information regarding various tasks that occur in the brain. In the medical field, EEGs are primarily used for various diagnoses, such as cerebrovascular or structural brain diseases, dementia, epilepsy, disturbances of consciousness, brain death, and other psychological disorders. However, EEGs are also used to investigate electrical brainwave responses [32, 33], alpha waves occur when an individual is in a relaxed state, whereas beta waves occur during alert and focused states [21]. Increased alpha brain activity is connected more to a relaxed state than to a condition of stress [34]. Recent advancements in cognitive neuroscience, information technology, and other invasive or noninvasive brain-signal capturing devices allow us to directly explore the human brain [35]. The NeuroSky Mind Wave is an alternative nonmedical instrument that was designed by the NeuroSky Company and can be used as a computerhuman interface to record human brainwave activity. The brain-computer interface (BCI) system is a direct communication pathway between the brain and an external device [36]. This technology is used in various computer-supported applications, such as video games, research software, and human health perspectives. The NeuroSky Mind Wave contains a single recording probe, and the signals are typically received through an electrode that is placed on the left side of the forehead above the eyes (Fp1 position) [37]. Compared with other professional instruments, this device is simple to operate, inexpensive, and has $86 \%$ accuracy. The major components that are built into the Mind Wave headset include a Bluetooth with a computer and a Think Gear generic chip, which contains signal data that can be visualized by a computer after processing. The NeuroSky Mind Wave headset provides unfiltered brainwave data and can be used by scientists to perform their own measurements. There is a little EEG information on the positive effects of horticultural activity in modern adults. Therefore, the goals of this study were to investigate the psychophysiological effects of horticultural activity in adults.

\section{Materials and Methods}

3.1. Participants. 40 Chinese female students (mean age $22.2 \pm 0.9$ years; mean weight $52.76 \pm 9.80 \mathrm{~kg}$; mean height $160.68 \pm 5.87 \mathrm{~cm}$ ) at the Sichuan Agricultural University in China participated in the experiment. None of the subjects had a history of psychological disorders. All drugs were strictly prohibited throughout the experiment. The experiments were performed in silence in a laboratory room at the College of Landscape Architecture. Prior to the experiments, the participants were fully informed of the methods of this study, and written informed consent was obtained. This experiment was conducted with the approval of the Ethics Committee of the College of Landscape Architecture, at the Sichuan Agricultural University, China.

3.2. Materials. Arranging flower baskets is a typical horticultural activity and was selected for this study; this activity involved the use of real flowers (i.e., roses (Rosa hybrid), chrysanthemums (Chrysanthemum morifolium), bamboos (Fargesia), lilies (Lilium candidum), and carnations (Dianthus caryophyllus) of approximately the same size and weight. A basic method regarding the making of flower baskets was taught to each participant prior to the experiments so that they could work more smoothly.

3.3. Protocol. The subjects were randomly divided into two groups. On the first day of the experiment, group A performed the horticultural activity (making flower baskets) by using real flowers, and group B performed the control activity (downloading research papers) by using a computer; the control activity is a typical computer activity, that also needs constant physical activity, similar to the horticultural task (Figure 1). On the second day, the groups switched activities. Both activities were performed in a seated position for $12 \mathrm{~min}$. 


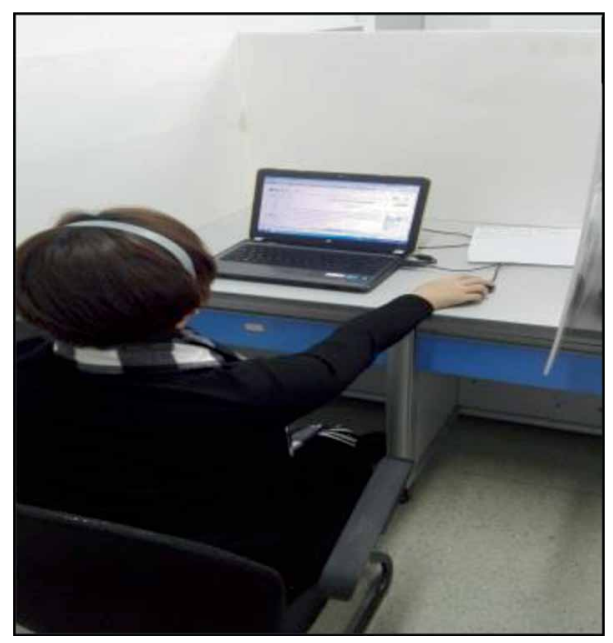

(a)

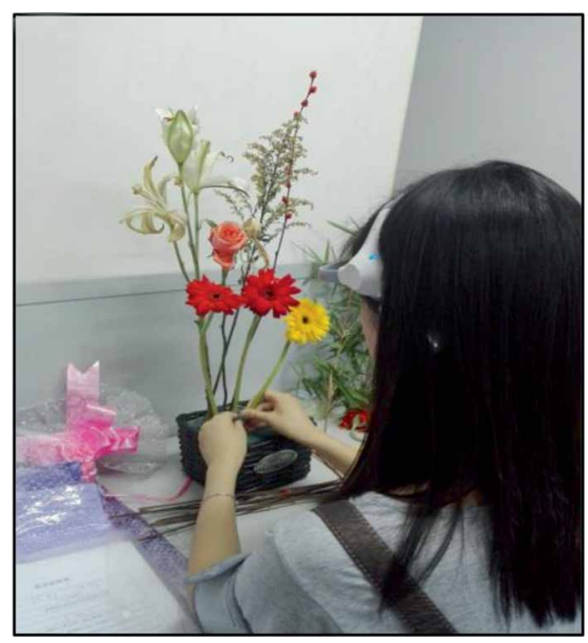

(b)

FIGURE 1: Photographs of experiments (a) a subject doing control activity (b) a subject doing horticultural activity.

3.4. Measurements. An EEG headset was attached to the subjects' heads in the waiting room. Then, the subjects entered the experimental room. After a 5-min rest in a seated position, the subjects performed their assigned tasks, i.e., either the horticultural activity that uses real flowers or the computer task, for $12 \mathrm{~min}$. EEGs were continuously measured during each task with a Mind Wave EEG headset (NeuroSky Mind Wave Beijing Oriental Creation Technology Co., LTD, China). Typically, an EEG records the brainwave activity inside the brain from the Fp1 position above the eye, which accords with the American Electroencephalographic Society's (1994) 10-20 system of electrode placement. In addition, the EEG setup consisted of four essential parts, namely, (1) a headband, (2) an ear-clip, (3) a sensor arm that contains the EEG electrode, and (4) a Bluetooth device. The Blood pressure and the pulse rate data were recorded before and after the tasks, by using a digital blood pressure device (Omron, HEM-7011, China-7201 type upper arm type intelligent electronic sphygmomanometer). The EEG data were recorded before and throughout the experiment (12 $\mathrm{min})$, and the alpha and beta brainwaves were compared between the two conditions to determine the participant's relaxation and attention levels in the two conditions. The measurements were obtained with a frequency of $512 \mathrm{~Hz}$, which means that values were obtained every second. The raw EEG data that include high alpha and high beta brainwaves were collected at 1-minute intervals at each experimental site and were compared between the two conditions. The higher values of experimental data show the participants in a more relaxed and alert condition. Relaxed wakefulness is a condition that is used for alpha activity during its predominant state [38]. The feelings that the participants experienced during the experiment were examined by using the State-Trait Anxiety Inventory (STAI) [39], a self-rated questionnaire that was completed before and after the tasks. The (STAI) consists of twenty questions (e.g., "I feel nervous"; "I feel relaxed"; "I feel frightened", etc.). The participants answered each of the twenty items according to how they felt before and after performing both activities by using the following four-point scale: $1=$ moderately, $2=$ not at all, $3=$ very much, and $4=$ somewhat.
3.5. Statistical Analysis. Statistical analyses were conducted by using SPSS 16.0 (SPSS Inc., Chicago, IL, USA). A paired $t$-test was used to determine the blood pressure differences between the two groups. A one-way analysis of variance (ANOVA) was used to compare the horticultural and control groups separately to study the significant differences in the pre- and posttests' alpha and beta mean values. A two-way repeated measure ANOVA was used to check whether there were any statistically significant differences between the horticultural and control groups' pretests and posttests changes in the alpha and beta mean values. For the physiological data, statistical significance was defined as $P<0.05$. For the psychological data, a Wilcoxon signed-rank test was used, and statistical significance was defined as $P<0.01$.

\section{Results}

Significant differences $(P<0.05)$ were observed in the systolic and diastolic blood pressure $(\mathrm{mmH}$ between the horticultural and control groups $(109.6 \pm 6.8 ; 116.8 \pm 11.8 ; \quad P=0.027$; $69.4 \pm 5.3 ; 72.8 \pm 6.3 ; P=0.058)$. However, no significant difference was found in the pulse rate (BPM) between the two groups $(74.0 \pm 7.2 ; 75.0 \pm 8.5 ; P=0.067)$ (Figure 2). No significant differences were observed between the horticultural group and the control group in the participants' relaxation, based on a one-way ANOVA that compared the alpha mean values between the groups on the pretest $(F=1.21, \quad P=0.22)$. However, the results of the posttest indicated that the control group had a drop in the participants' relaxation based on the alpha mean values. Furthermore, a one- way ANOVA indicated that this drop in the alpha mean values by the control group over the pre- and posttests' duration was significant $(F=2.84$, $P<0.01)$. In contrast, the horticultural group had a significant increase in the participants' relaxation, based on a one-way ANOVA of the alpha mean values over the pre- and posttest duration $(F=2.02, P=0.0038)$.

However, a two-way ANOVA indicated that the pre- and posttests variations in the participants' relaxation, based on 


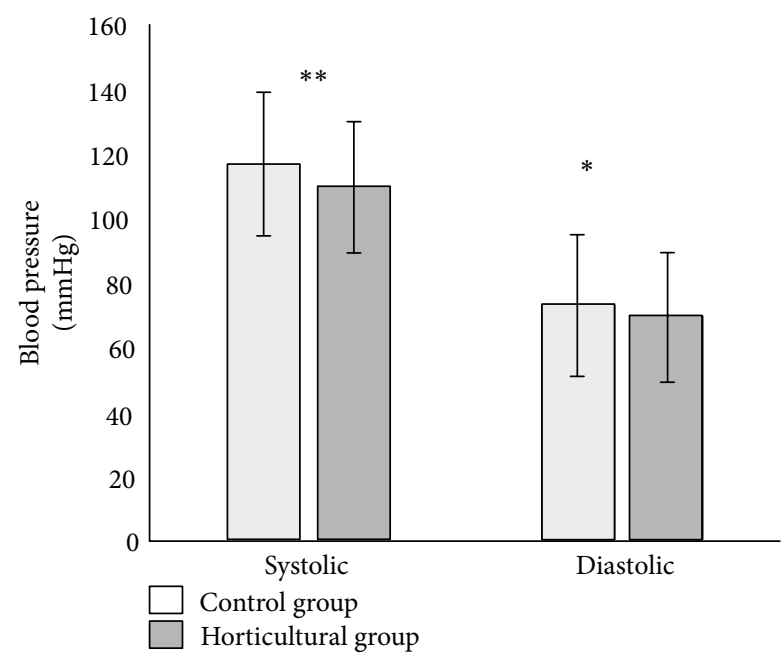

Figure 2: Comparison of blood pressure data between the horticultural group and control group. $N=40$ : mean \pm SE. ${ }^{* *} P<0.01$ : determined using a paired $t$-test.

the alpha mean values, for the horticultural group were significantly greater than the pre- and posttests variations in the participants' relaxation, based on the alpha mean values, for the control group $(F=16.2, P<0.01)$. Similarly, no significant differences were observed between the horticultural group and the control group in the participants' attention, based on a one-way ANOVA that compared the beta mean values between the groups on the pretest $(F=1.13, P=0.30)$. Moreover, the results of the posttest indicated that the control group had a drop in the participants' attention based on the beta mean values. Furthermore, a one-way ANOVA indicated that this drop in the beta mean values by the control group over the pre- and posttests' duration was not significant $(F=0.89$, $P=0.61$ ). Furthermore, the results of the posttest indicated that the horticultural group had an increase in the participants' attention based on the beta mean values. However, based on a one-way ANOVA, the horticultural group beta mean values over the pre- and posttest durations were not significant $(F=0.81, P=0.72)$. A two-way ANOVA indicated that the pre- and posttests variations in the participants' attention, that were based on the beta mean values, for the horticultural group were significantly greater than the pre- and posttests variations in the participants' attention, that were based on the beta mean values, for the control group $(F=13.01$, $P=0.001)$. According to the STAI data, the subjects' elings during the horticultural task (which used real flowers) were different from their feelings during the control task (computer task). There were significant differences between the two tasks in the total anxiety score, which was measured before and after the completion of the 12-min. tasks. As shown in Figure 3, the level of anxiety after the horticultural activity was significantly lower than the level of anxiety after the control activity (Horticultural group; $38.5 \pm 4.5$; Control group; $44.4 \pm 4.4$; $P=0.001$. However, there was no significant difference in the baseline (before the activities), which suggests that the horticultural activity may have a better effect in lowering anxiety in adults.

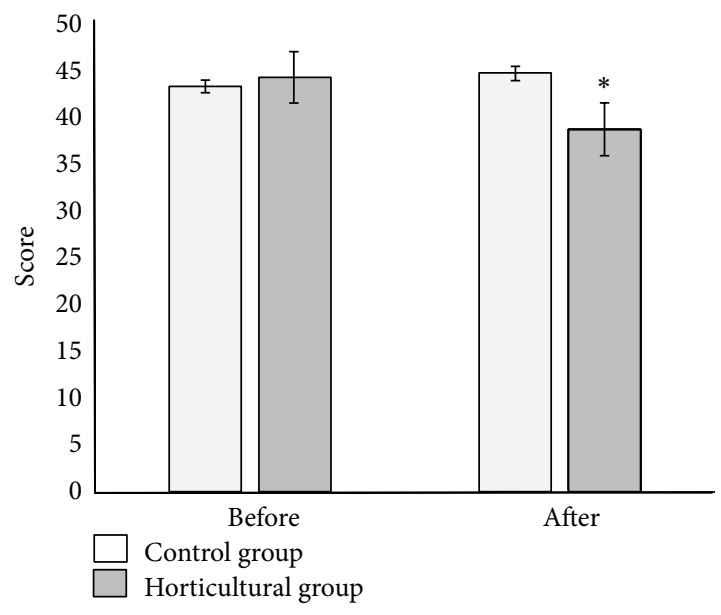

FIgURE 3: Comparison of pretest/posttest STAI data between the horticultural group and the control group. $N=40$ : mean $\pm \mathrm{SE}$. ${ }^{*} P<0.01$ : determined using a paired $t$-test.

\section{Discussion}

In this experiment, we investigated the relaxation effects of a horticultural activity by measuring and comparing the participants' psychological and physiological responses between two tasks: a flower basket making activity by using real flowers and a computer task. Blood pressure was significantly decreased in the horticultural activity than in the control activity. The lower blood pressure indicates that interaction with plants has a beneficial effect on adults. Studies on human-plant interaction have indicated that direct or indirect contact with plants or natural surroundings can have a greater beneficial effect on people's well-being [5, 10, 40]. A comparison of blood pressure finds that the horticultural activity has a positive effect on mental stress that increases when a human is exposed to a stressor. The findings of this experiment agree with a previous study that reported that plant activity reduces stress [41]. The findings of the EEGs show that plants have a greater relaxing effect on the brain by enhancing brain activity, which changes when a person connects to a stressor. In this experiment, the value of the alpha brainwaves increases after the horticultural activity, while a lower alpha brainwave value is observed after the control activity. The increase in alpha brainwaves clearly shows that the participants were mentally relaxed; however, lower alpha brainwaves indicated the condition of stress. Studies on EEGs have reported that an increase in mental workload caused the alpha waves to decrease or disappear [42]. Additionally, alpha waves are associated with relaxation and mental coordination $[43,44]$. Previous plant studies with EEGs have reported that an increase of satisfaction or relaxation causes increased alpha and beta brainwave activity [45]. Moreover, another experiment regarding horticultural activities with and without plants reported that the ratio of both the alpha and beta brainwaves significantly increased when subjects closed their eyes only in the plant group [46]. Thus, plants have strong effects on the brain by changing the brainwave activity. Furthermore, the value of the beta brainwaves increases after the horticultural activity, while lower beta brainwaves are observed after the control activity. 
The increase in beta brainwaves clearly shows that the participants were highly alert; however, lower beta brainwaves indicate a lack of attentiveness or stress. Increases in beta waves correlate with a highly alert state of mind, and decreases correlation with a drowsiness state [47]. A study by Monina Islarm reported that an EEG could be used to determine emotions. In his study, both the alpha and beta waves are active during a workload, and when the workload on the brain increases, beta wave activity then increases. The study shows that when people use more focus on a specific task, the ratio of the beta power increases [48]. Additionally, beta waves are fast wave activity and are used to engage in decision making and mental tasks [49] such as attending lectures in a classroom or solving problems. Both alpha and beta waves naturally appear during the active and resting conditions. Our behavior, emotions, and thoughts are the impressions of neuronal activity inside the brain. The appearance of brain waves shows the neuronal electrical activity within the brain. EEGs record these electrical fluctuations and represent them as waves. Brain waves with a slower frequency range appear when we feel dreamy or tired. In contrast, higher frequencies are dominant when we feel excited or alert [50]. Finally, the STAI results also show that the control activity may have negative effects on the mood state. The results show that the participants felt more stress after performing the control activity than after performing the horticultural activity. Studies have reported that active interaction with plants has positive effects on mood $[51,52]$.

\section{Conclusion}

The findings of this experiment suggest that horticultural activity lowers blood pressure and, increases alpha and beta brainwave activity as compared with mental task. Future studies could determine whether the positive effects of horticultural activity last over longer time periods with more diversified groups with large sample. Furthermore, a comparison of horticultural therapy with a pet therapy (control group) could help researchers better understand the effectiveness of plants and should be tested in future studies.

\section{Data Availability}

The research data used to support the findings of this study are available from the corresponding author upon request.

\section{Ethical Approval}

Subject's written informed consent was obtained. This study arranged with the approval of the local Ethics Committee College of Landscape Architecture, Sichuan Agricultural University, China.

\section{Consent}

Subject's written informed consent was obtained for the publication of images.

\section{Conflicts of Interest}

Authors declare that they have no conflicts of interests.

\section{Authors' Contributions}

Jiang Tao and Ahmad Hassan worked equally as a first author and were responsible for data obtaining, statistical inquiry and article development. Prof. Chen Qibing and prof. LIU YINGGAO supervised the experiment. Guo Li, Mingyan Jiang, Deng Li, Li Nian, Lv Bing-Yang, Zhong Ziqin helped during the experiment. All authors read and approved the final manuscript.

\section{Acknowledgments}

In addition, we appreciate the help of Shu Li, Li Yuting Junzhuo Li, Shang guan Ziyue, Cheng Xinyi Wang Yiyan, Muhammad Tahir, Muhammad Sohaib Tahir and Muhammad Salman Tahir for assistance. This study was sponsored by the National Natural Science Foundation of China (No. 31570700), the Guizhou university high level scientific research foundation for the introduction of talent (No. 2018-50), and the National Key R\&D Program of China (2018YFD0600105).

\section{References}

[1] E. Friedmann and S. A. Thomas, "Pet ownership, social support, and one-year survival after acute myocardial infarction in the cardiac arrhythmia suppression trial (CAST)," The American Journal of Cardiology, vol. 76, no. 17, pp. 1213-1217, 1995.

[2] H. Frumkin, "Beyond toxicity: human health and the natural environment," American Journal of Preventive Medicine, vol. 20, no. 3, pp. 234-240, 2001.

[3] A. H. Katcher and A. M. Beck, "Health and caring for living things," Anthrozoös, vol. 1, no. 3, pp. 175-183, 1987.

[4] C. Maller, M. Townsend, A. Pryor, P. Brown, and L. St Leger, "Healthy nature healthy people: "contact with nature" as an upstream health promotion intervention for populations," Health Promotion International, vol. 21, no. 1, pp. 45-54, 2006.

[5] R. Kaplan, "Impact of urban nature: a theoretical analysis," Urban Ecology, vol. 8, no. 3, pp. 189-197, 1984.

[6] R. S. Ulrich, "Visual landscapes and psychological well-being," Landscape Research, vol. 4, no. 1, pp. 17-23, 1979.

[7] R. S. Ulrich, R. F. Simons, B. D. Losito, E. Fiorito, M. A. Miles, and M. Zelson, "Stress recovery during exposure to natural and urban environments," Journal of Environmental Psychology, vol. 11, no. 3, pp. 201-230, 1991.

[8] R. S. Ulrich, "Response to natural," Behavior and the Natural Environment, vol. 6, p. 85, 1983.

[9] R. S. Ulrich, "Human responses to vegetation and landscapes," Landscape and Urban Planning, vol. 13, pp. 29-44, 1986.

[10] R. S. Ulrich and R. F. Simons, Eds., Recovery from Stress during Exposure to Everyday Outdoor Environments, Proceedings of EDRA, 1986. 
[11] M. L. Bennett, "The relation between landscape type and perceived restorative character of coastal landscapes," The University of Utah, 2011.

[12] R. S. Ulrich, "Influences of passive experiences with plants on individualwell-beingandhealth," TheRoleofHorticultureinHuman Well-being and Social Development, pp. 93-105, Timber Press, Portland, Oregon, 1992.

[13] R. Kaplan and S. Kaplan, The Experience of Nature: A Psychological Perspective, CUP Archive, 1989.

[14] P. H. Kahn Jr, R. L. Severson, and J. H. Ruckert, "The human relation with nature and technological nature," Current Directions in Psychological Science, vol. 18, no. 1, pp. 37-42, 2009.

[15] M. J. Molina and L. T. Molina, "Megacities and atmospheric pollution," Journal of the Air \& Waste Management Association, vol. 54, no. 6, pp. 644-680, 2004.

[16] A. M. Breland-Noble, C. Bell, and G. Nicolas, "Family first: the development of an evidence-based family intervention for increasing participation in psychiatric clinical care and research in depressed african American adolescents," Family Process, vol. 45 , no. 2, pp. 153-169, 2006.

[17] C. Brod, Technostress: The Human Cost of the Computer Revolution, Addison Wesley Publishing Company, 1984.

[18] M. K. Honeyman, "Vegetation and stress: a comparison study of varying amounts of vegetation in countryside and urban scenes," The Role of Horticulture in Human Well-being and Social Development, pp. 143-155, Timber Press, Portland, Oregon, 1992

[19] G. G. Fried and M. J. Wichrowski, "Horticultural therapy: a psychosocial treatment option at the Stephen D. Hassenfeld children's center for cancer and blood disorders," Primary Psychiatry, vol. 15, no. 7, 2008.

[20] M. Adachi, C. Rohde, and A. Kendle, "Effects of floral and foliage displays on human emotions," HortTechnology, vol. 10, no. 1, pp. 59-63, 2000.

[21] C.-Y. Chang and P.-K. Chen, "Human response to window views and indoor plants in the workplace," HortScience, vol. 40, no. 5, pp. 1354-1359, 2005.

[22] C. K. Coleman and R. K. Mattson, "Influences of foliage plants on human stress during thermal biofeedback training," HortTechnology, vol. 5, no. 2, pp. 137-140, 1995.

[23] E. Kim and R. Mattson, "Stress recovery effects of viewing redflowering geraniums," Journal of Therapeutic Horticulture, vol. 13, pp. 4-12, 2002.

[24] C. A. Shoemaker, K. Randall, P. D. Relf, and E. S. Geller, "Relationships between plants, behavior, and attitudes in an office environment," HortTechnology, vol. 2, no. 2, pp. 205-206, 1992.

[25] T. Fjeld, "The effect of interior planting on health and discomfort among workers and school children," HortTechnology, vol. 10, no. 1, pp. 46-52, 2000.

[26] T. Fjeld, B. Veiersted, L. Sandvik, G. Riise, and F. Levy, “The effect of indoor foliage plants on health and discomfort symptoms among office workers," Indoor and Built Environment, vol. 7, no. 4, pp. 204-209, 1998.

[27] T. Fjeld, F. Levy, and C. Bonnevie, "Foliage plants both with or without additional full-spectrum fluorescent light, may reduce in-door health-and discomfort complaints," Proceedings Indoor Air, vol. 99, no. 4, pp. 616-621, 1999.
[28] H. Anupama, N. Cauvery, and G. Lingaraju, "Brain computer interface and its types-a study," International Journal of Advances in Engineering \& Technology, vol. 3, no. 2, p. 739, 2012.

[29] N. V. Thakor and S. Tong, "Advances in quantitative electroencephalogram analysis methods," Annual Review of Biomedical Engineering, vol. 6, no. 1, pp. 453-495, 2004.

[30] Y. Yao, Z. Lian, W. Liu, and Q. Shen, "Experimental study on physiological responses and thermal comfort under various ambient temperatures," Physiology \& Behavior, vol. 93, no. 1, pp. 310-321, 2008.

[31] A. F. Jackson and D. J. Bolger, "The neurophysiological bases of EEG and EEG measurement: a review for the rest of us," Psychophysiology, vol. 51, no. 11, pp. 1061-1071, 2014.

[32] K. Sowndhararajan, M. Seo, M. Kim, H. Kim, S. Kim, "Effect of essential oil and supercritical carbon dioxide extract from the root of Angelica gigas on human EEG activity". Complementary Therapies in Clinical Practice, vol. 28, pp. 161-168, 2017.

[33] Y. Sugawara, C. Hara, T. Aoki, N. Sugimoto, and T. Masujima, "Odor distinctiveness between enantiomers of linalool: difference in perception and responses elicited by sensory test and forehead surface potential wave measurement," Chemical Senses, vol. 25, no. 1, pp. 77-84, 2000.

[34] R.H.FazioandJ.Cooper, "Arousalin thedissonanceprocess," Social Psychophysiology: A Sourcebook, pp. 122-152, Guilford Press, New York, 1983.

[35] D. Wijayasekara and M. Manic, Eds., "Human machine interaction via brain activity monitoring", in 2013 The 6th International Conference on Human System Interaction (HSI), IEEE, Sopot, Poland, 2013.

[36] J. R. Wolpaw, N. Birbaumer, W. J. Heetderks et al., "Braincomputer interface technology: a review of the first international meeting," IEEE Transactions on Rehabilitation Engineering, vol. 8, no. 2, pp. 164-173, 2000.

[37] R. Robbins and M. Stonehill, "Investigating the NeuroSky MindWave $^{\mathrm{Tm}}$ EEG Headset," Transport Research Foundation, pp. 1-28, 2014.

[38] J. T. Cacioppo, L. G. Tassinary, and G. G. Berntson, "Psychophysiological science," Handbook of Psychophysiology, vol. 2, pp. 3-23, 2000.

[39] N. Hidano, M. Fukuhara, M. Iwawaki, S. Soga, and C. Spielberger, State-trait Anxiety Inventory-Form JYZ, Japan UNI Agency (in Japanese), Tokyo, 2000.

[40] R. S. Ulrich, "View through a window may influence recovery from surgery," Science, vol. 224, no. 4647, pp. 420-421, 1984.

[41] M.-S. Lee, J. Lee, B.-J. Park, and Y. Miyazaki, "Interaction with indoor plants may reduce psychological and physiological stress by suppressing autonomic nervous system activity in young adults: a randomized crossover study," Journal of Physiological Anthropology, vol. 34, no. 1, p. 21, 2015.

[42] S. Miller, Workload Measures, National Advanced Driving Simulator Iowa City, United States, 2001.

[43] E. Başar, "A review of alpha activity in integrative brain function: fundamental physiology, sensory coding, cognition and pathology," International Journal of Psychophysiology, vol. 86, no. 1, pp. 1-24, 2012.

[44] S. C. Kim, M. H. Lee, C. Jang, J. W. Kwon, and J. W. Park, "The effect of alpha rhythm sleep on EEG activity and individuals' attention," Journal of Physical Therapy Science, vol. 25, no. 12, pp. 1515-1518, 2013. 
[45] J. Qin, C. Sun, X. Zhou, H. Leng, and Z. Lian, "The effect of indoor plants on human comfort," Indoor and Built Environment, vol. 23, no. 5, pp. 709-723, 2014.

[46] K. Yamane, M. Kawashima, N. Fujishige, and M. Yoshida, Eds., "Effects of interior horticultural activities with potted plants on human physiological and emotional status," XXVI International Horticultural Congress: Expanding Roles for Horticulture in Improving Human Well-Being and Life Quality, vol. 639, ISHS Acta Horticulturae, 2002.

[47] B.-G. Lee, B.-L. Lee, and W. Y. Chung, "Mobile healthcare for automatic driving sleep-onset detection using wavelet-based EEG and respiration signals," Sensors, vol. 14, no. 10, pp. 1791517936, 2014.

[48] M. Islam and M. Ahmad, "Modeling of human emotion with effective frequency band during a test of sustained mental task," Electrical and Computer Engineering (WIECON-ECE), in IEEE International WIE Conference, IEEE, 2015

[49] C. Neuper and G. Pfurtscheller, "Event-related dynamics of cortical rhythms: frequency-specific features and functional correlates," International Journal of Psychophysiology, vol. 43, no. 1, pp. 41-58, 2001

[50] G. Buzsáki and B. O. Watson, "Brain rhythms and neural syntax: implications for efficient coding of cognitive content and neuropsychiatric disease," Dialogues in Clinical Neuroscience, vol. 14, no. 4, p. 345, 2012.

[51] A. Dravigne, T. M. Waliczek, R. Lineberger, and J. Zajicek, "The effect of live plants and window views of green spaces on employee perceptions of job satisfaction," HortScience, vol. 43, no. 1, pp. 183-187, 2008.

[52] V. I. Lohr and C. H. Pearson-Mims, "Children's active and passive interactions with plants influence their attitudes and actions toward trees and gardening as adults," HortTechnology, vol. 15, no. 3, pp. 472-476, 2005. 